\title{
Transmitting audio via fiber optics under nonlinear effects and optimized tuning parameters based on co-simulation of MATLAB and OptiSystem ${ }^{\text {TM }}$
}

\author{
Abdulrasul A. Al-Hayder, H. J. Abd, Ahmed Samawi Alkhafaji \\ Department of Electrical Engineering, College of Engineering, Babylon University, Iraq
}

\begin{tabular}{l}
\hline \hline Article Info \\
\hline Article history: \\
Received Apr 17, 2019 \\
Revised Nov 16, 2019 \\
Accepted Jan 8, 2020 \\
\hline
\end{tabular}

\section{Keywords:}

Audio signal

Nonlinear effect

NRZ

Q-factor

Raised cosine

\begin{abstract}
The ability of fiber optic to overcome the signal transmission problems is making it a dominant transmission medium. Despite of this major positive attribute of optic fibers, there is still a downside for using the fiber optic communication; that is the nonlinearity problem. For the first time, a design of an audio signal is suggested and executed in MATLAB with integration with OptiSystem ${ }^{\mathrm{TM}}$ Software .The audio signal then transmitted in different shapes of modulation signals (NRZ, RZ, \& RC) for different distances $(100 \mathrm{~km} \& 75 \mathrm{~km})$ via a fiber optic media to be received in a receiving part of the simulated system. Three tests are used to do so. The first is the Quality-facto (Q-Factor) against the received power, second test is eye diagram performance and finally is the measuring of the amplitude of output (received) signal for each modulation signal shape using the Oscilloscope Visualizer. The NZR modulation signal was found to be the best one of the three used signals' types in all three tests. The Q-factor for NRZ pulse shape $(=12)$ was higher than that for $\mathrm{RZ}(=10)$ and $\mathrm{RC}(=8)$ for a $100 \mathrm{~km}$ distance at the same received power level.
\end{abstract}

Copyright $\odot 2020$ Institute of Advanced Engineering and Science. All rights reserved.

\section{Corresponding Author:}

H. J. Abd,

Department of Electrical Engineering, College of Engineering,

Babylon University,

Babil-Hilla, 40 street- center of the city of Hilla, Iraq.

Email: haiderlaser@yahoo.com

\section{INTRODUCTION}

Since the optical fiber, as a communication medium, is providing a lot more bandwidth than the copper capacity, it is become the main communication systems medium [1-4]. It is offering low loss over a high bandwidth, reduction of unwanted alterations, no electromagnetic intervention, and its long life. Optical fiber and wireless communications are almost complementary, yet, especially at gigabits per second transmission rates, wireless network having some hindrance to accomplish high end-to-end data delivery performance. Optical fibers are widely used in communication, sensors, lighting and other usage [5-6]. In communication, optical fibers are providing a long distance and a higher data rates transmission comparing to other forms. Using copper wires in communication is less efficient than the optical fibers in long distances transmission besides the better immunity that the optical fibers provide against the electromagnetic interference. Optical fibers also provide a 100\% signal security comparing to the copper cables [7-10]. The fibers are not emitted the transmitted signals without tampering while the copper cable dose, this fiber property preventing the drawing of transmitted signal. The non-linear behavior of optical fiber is its major weakness [11-20]. This nonlinearity is a directly proportional with the power of the signal been transmitted through the fiber optic. At the increasing of the light power, the nonlinearity behavior is becoming out of control and the probability of both distorting the transmitted signal and degrading the system efficiency may be raised [21-22]. There is also a higher chance of a signal interference as a result 
of the non-linearity. When these signals are transmitted in an equally spaces' separated channels will create what is called the four-wave mixing (FWM). Suppressing the FWM in optical communication is improving the system efficiency, which is a high priority goal. Several techniques been done to achieve an effective reduction of FWM. Wavelength swept WDM, the allocation of polarization and effective frequency, WDM/TDM (wavelength/time division multiplexing), and non-uniform spacing of channels ware applied [23-30]. These techniques are needing an extra complexity in system design or at least needing a compensator to improve the signal dispersion. Some of these techniques are negatively effecting the WDM capacity in order to compress the FWM.

The modulation technique used is influencing the signal's interference, which is occurring when FWM is used. The transmission of audio signal via optical fiber under the non-linear conduct is not yet investigated. The goal of this paper is to study the transmission of audio signal via fiber optic at a non-linear state and different modulation signals to find the best one for satisfied system performance. For this purpose, a system to simultaneously transmitting and receiving an audio signal via a fiber optic cable at its non-linear behavior is designed and implemented using the MATLAB and OptiSystem simulation model. The proposed system performance is tested for different types of modulation signals to get the best one.

\section{MODELING OFAUDIO SIGNAL AND SIMULATION SYSTEM DESIGN}

The system that designed for this work is highlighted in this section. It is simulated using MATLAB integrated with OptiSystem ${ }^{\mathrm{TM}}$. The simulated system of this work was designed and studied in three steps. The first is designing and implementing the new audio communication signal in MATLAB and integrated it into the OptiSystem ${ }^{\mathrm{TM}}$. The audio signal of the parameters shown in Table 1 then simulated with the fiber link as a second step. The third step is to study the performance of the optical signal under two transmission lengths $(100 \mathrm{~km}$ and $75 \mathrm{~km})$ for different pulse shapes modulation signals such as Non-Return-Zero (NRZ), Return-Zero (RZ) and RC (Raised Cosine). The design of the transmitter and receiver of the model is shown in Figures 1 and 2.

The transmitter part consists of an array of a continuous-wave $(\mathrm{CW})$ produced by laser sources connected to an external modulator. The channel frequency is set to $193 \mathrm{THz}$. The external modulator is consisting of a Pseudo-Random Bit Sequence (PRBS) generator connected to a pulse generator to modulate the optical signals using different pulse shapes (NRZ, RZ, and RC) modulation signals which is connected to Mach-Zehnder modulator (MZM) acting as an intensity modulator. A single mode fiber is used as an optical link.

At the receiver, the de-multiplexer splits the collected frequencies. The PIN photo diode is detecting the signal with a responsively $(\mathfrak{R})$ of $0.8 \mathrm{~A} / \mathrm{W}$ and a dark current of $10 \mathrm{nA}$. The signal is then passed through the low-pass Bessel filter, which is also linked, to the BER analyzer that is used to generate the graph. The system's parts (transmitter, optical channel and receiver) are shown in Figures 1 and 2, and it is parameters are explained in Table 2.

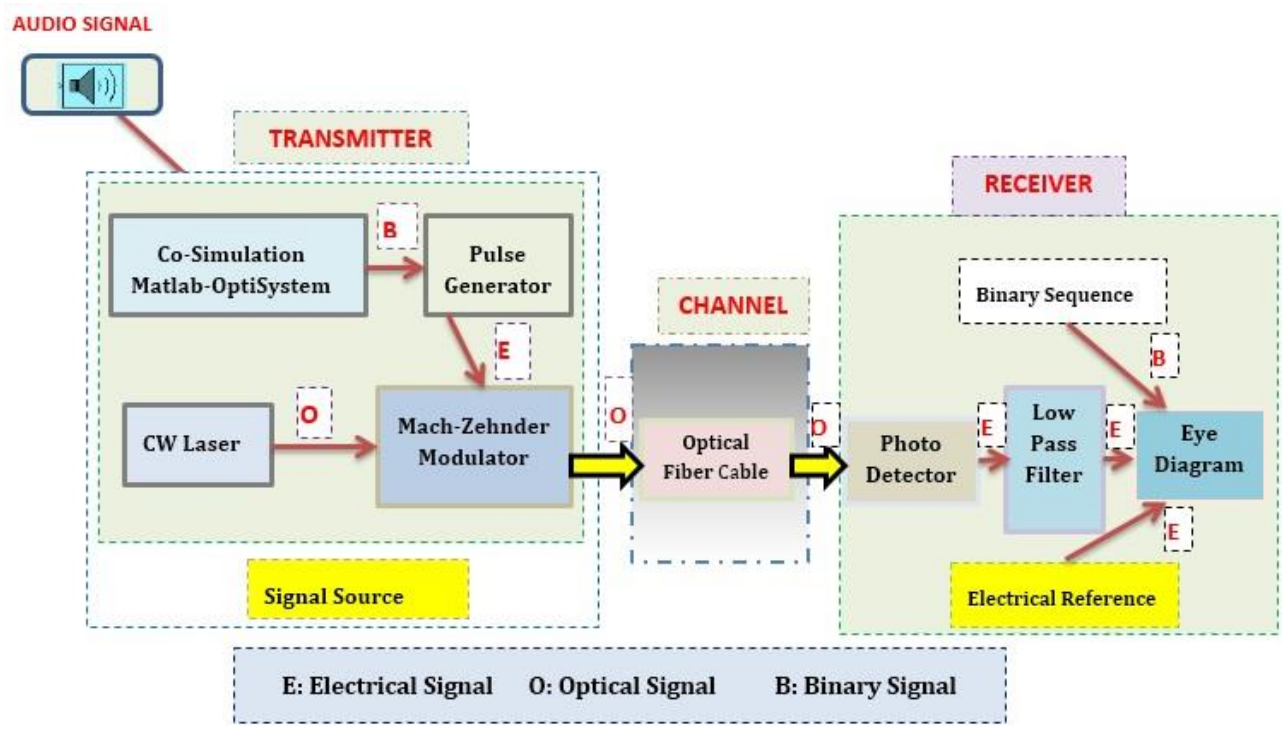

Figure 1. Full circuit diagram 


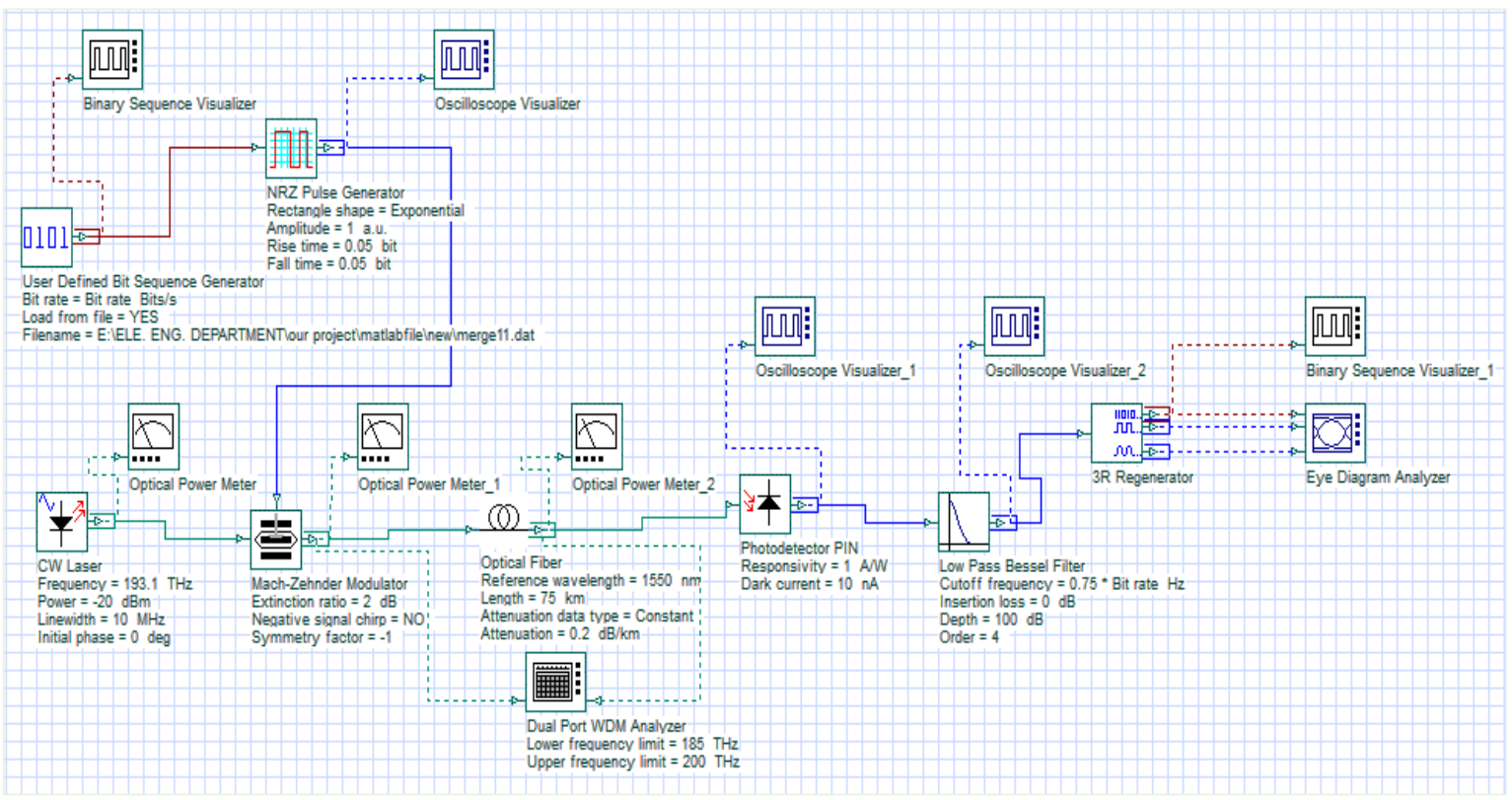

Figure 2. Optical simulation system design

Table 1. Audio signal properties

\begin{tabular}{|c|c|c|}
\hline Parameter & Unit & Values \\
\hline File Format & Wave Sound (.wav) & - \\
\hline filename & story.wav & - \\
\hline Bit Rate, B & kbps & 141 \\
\hline Number of channels & $\mathrm{n}$ & 2 \\
\hline Sample Rate, Fs & $\mathrm{Hz}$ & 44100 \\
\hline Queue duration & Seconds & 10 \\
\hline Frame size & Sample & 1024 \\
\hline Output data type & double & - \\
\hline Channel type & - & Stereo \\
\hline load file to OptiSystem ${ }^{\mathrm{TM}}$ & mat & \\
\hline Type of media path & Online media & - \\
\hline
\end{tabular}

Table 2. System parameters

\begin{tabular}{lcc}
\hline Parameter & Unit & Values \\
\hline Fiber length, $\mathrm{L}$ & $\mathrm{km}$ & $75-100$ \\
Input power, Pi & $\mathrm{dBm}$ & $(-20)$ to $(-12)$ \\
Input frequencies & $\mathrm{THz}$ & 193 \\
Dispersion, Dc & $\mathrm{ps} / \mathrm{nm} . \mathrm{km}$ & 17 for SMF \\
Dispersion slope S & $\mathrm{ps} / \mathrm{nm} 2 . \mathrm{km}$ & 0.075 for SMF \\
Cross effective area, Aeff & $\mu \mathrm{m} 2$ & 80 for SMF \\
Third order susceptibility, X111 & $\mathrm{m} 3 / \mathrm{w} . \mathrm{s}$ & $6 \times 10-15$ \\
Refractive index, $\mathrm{n}$ & - & 1.48 \\
Speed of light, c & $(\mathrm{m} / \mathrm{s})$ & $3 \times 108$ \\
Attenuation factor, & $(\mathrm{dB} / \mathrm{km})$ & 0.2 \\
Number of channels & - & 1 \\
Receiver noise temperature, Tn & $\mathrm{K}$ & 300 \\
Receiver load resistor, RL & $\Omega$ & 1030 \\
\hline
\end{tabular}

Schrödinger Equation (NLSE) is used to explain the envelope of the optical field when the nonlinear effects are in the form that is given by the following equation [12],

$$
\frac{\partial A(z, t)}{\partial z}+\frac{\alpha}{2} A(z, t)+B_{1} \frac{\partial A(z, t)}{\partial t}+\frac{j}{2} B_{2} \frac{\partial^{2} A(z, t)}{\partial t^{2}}-\frac{1}{6} B_{3} \frac{\partial^{3} A(z, t)}{\partial t^{3}}=-j \gamma|A(z, t)|^{2} A(z, t) .
$$

where $\mathrm{A}$, is the wave-field envelope, $\alpha$ is the fiber loss factor, B1, B2, and B3 are the dispersion factors and $\gamma$ is the nonlinear constant. 
To assess the system performance under the impact of nonlinear effect, which is the FWM crosstalk, and with other noise types, we should add the FWM crosstalk expressions as in (2),

$$
C_{I M}^{(m)}=\frac{1}{8} \sum_{I} \frac{P_{i j k}}{P_{s}}+\frac{1}{4} \sum_{I I I} \frac{P_{i i k}}{P_{s}}
$$

where $C_{I M}^{(m)}$ is the FWM crosstalk effective in intensity modulation-direct detection (IM-DD) transmission. Note that the probability of transmit bit " 1 " at any time for every user is $1 / 2$. Equation (3) explaining the relationship between the shot noise power (the left side) and the thermal noise power (the right side) [31]:

$$
\sigma^{2}=\frac{e B \Re P_{s r} W}{N}+\frac{4 K_{b} T_{n} B}{R_{L}}
$$

where, Psr is a received peak power of the signal light, B electrical bandwidth of the receiver, $\mathfrak{R}$ is the detector responsivity, e is the Electron's charge, Kb Boltzmann's constant, Tn Absolute receiver noise temperature, RL Receiver load resistor. The average SNR (4) is calculated from (2) and (3) [31]:

$$
S N R=\left[\frac{\left(\frac{\Re P_{s r} W}{N}\right)^{2}}{\frac{e B \Re P_{s r} W}{N}+\frac{4 K_{b} T_{n} B}{R_{L}}+2 K^{2} P_{s}^{2} C_{I M}}\right]
$$

Then BER is calculated in (5),

$$
B E R=P_{e}=\frac{1}{2} \operatorname{erfc}\left(\sqrt{\frac{S N R}{8}}\right),
$$

and (6) is to find the Q-factor,

$$
B E R=\frac{1}{2} \times \operatorname{erfc}\left[\frac{Q}{\sqrt{2}}\right]
$$

\section{RESULTS AND ANALYSIS}

This section is showing the finding of this work with analysis. The following results are obtained from the simulated system using transmission distances of $100 \mathrm{~km}$ and $75 \mathrm{~km}$ fiber length for 3-pulse's shapes NRZ, RZ, and RC for each distance:

\subsection{Results of proposed system design using $100 \mathrm{~km}$ fiber length \\ 3.1.1. Received power versus $Q$-factor}

The relationship between the Received power and Q-factor under the effect of nonlinearity for different pulse shaping is shown in Figure 3. The optical input power is started at $-20 \mathrm{dBm}$ and increased by a step of $1 \mathrm{dBm}$ till $-12 \mathrm{dBm}$ as a final input power. The obtained results are showing that the Q-factor is increased for the increasing in the received power for all shapes of pulses, however the system's behavior is different for different modulation signals under a nonlinear effect. The best value of Q-factor 12 was obtained when NRZ modulation signal is used at a received power of $(-35 \mathrm{dBm})$. In the case of the RC modulation signal, Q-factor is a minimum (only in the range of 8 ) at the same received power $(-35 \mathrm{dBm})$. For RZ pulses, the Q-factor is 10 which is in between the two previous values under the same conditions. This means that the NRZ gives a high resistance to the nonlinear effect in comparing with other two types.

\subsubsection{Eye diagram performance}

Figure 4 is showing the eye diagrams performances of the proposed system's approach taken at ch1 at Pin $=-14 \mathrm{dBm}$. It is clear that using NRZ pulses, shown in Figure 4(a), is having wider eye opening which means a better performance and a higher invulnerability to noise comparing to the other two modulating signals as shown in Figures 4(b) for RZ and 4(c) for RC modulation signals. 


\subsubsection{Signal output behavior}

Oscilloscope Visualizer is used to analyze and compare the electrical signals output for the $100 \mathrm{~km}$ fiber length of 3 types of modulation signals (NRZ, RZ, and RC). Figure 5 is showing that the optical system using NRZ, Figure 5(a), as a modulation signal is having higher power amplitude than the ones using RZ and $\mathrm{RC}$, Figure 5(b) and 5(c). This means that the signal's power is higher than the noise power and this shall make the system's output at the receiver is more reliable and efficient.

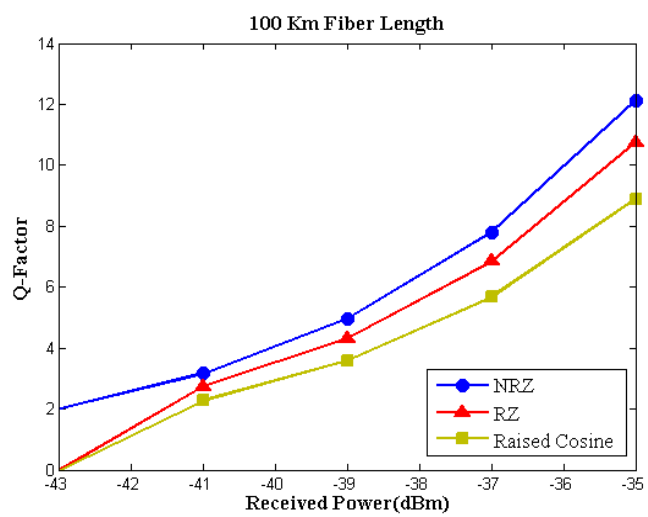

Figure 3. Q factor versus received Power at different pulse shape

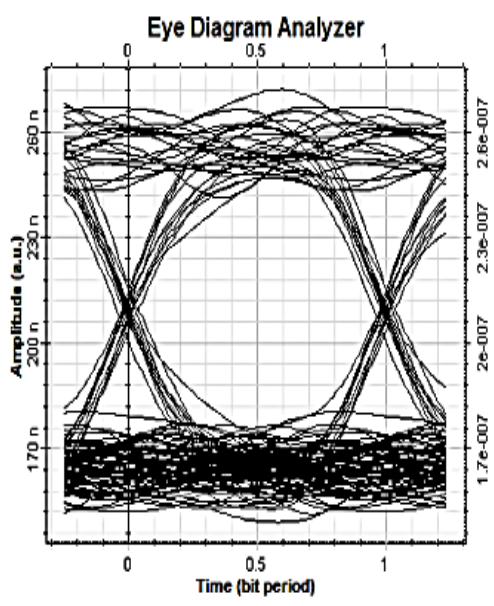

(a)

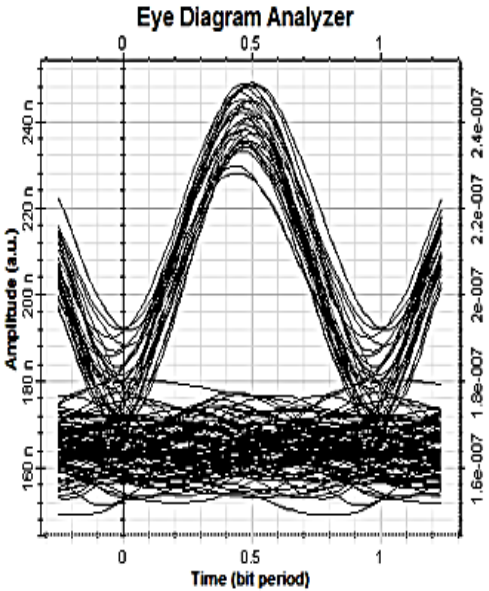

(b)

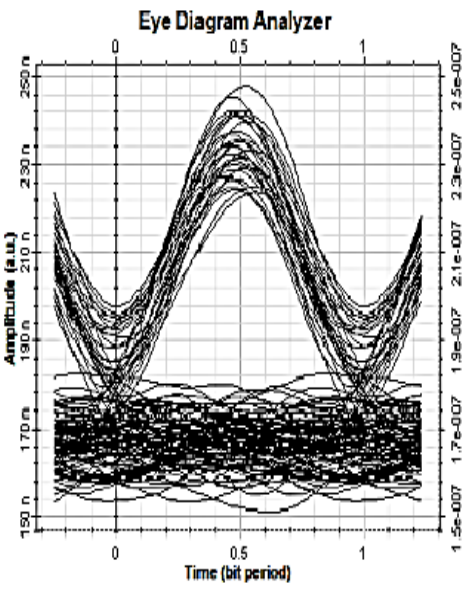

(c)

Figure 4. Eye diagram performance of $100 \mathrm{~km}$ length for (a) NRZ, (b) RZ, and (c) RC

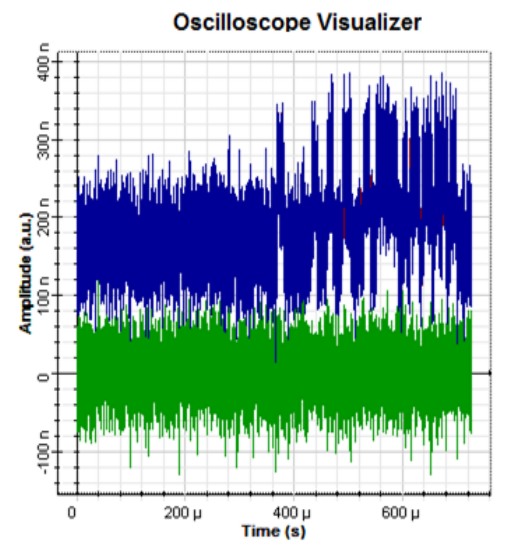

(a)

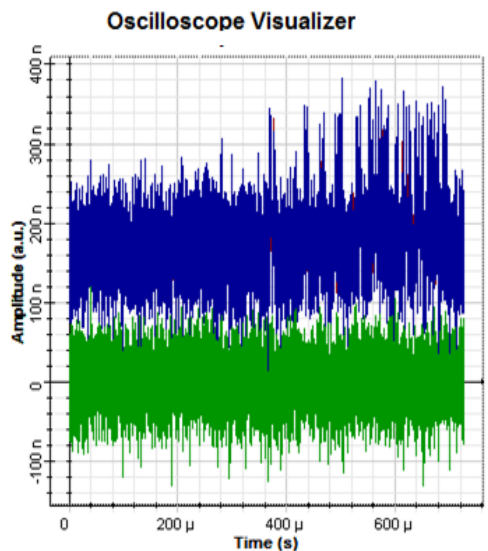

(b)

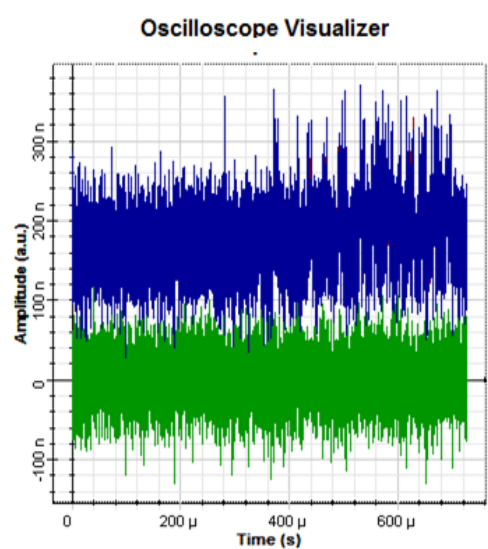

(c)

Figure 5. Signal output using oscilloscope visualizer of $100 \mathrm{~km}$ for (a) NRZ, (b) RZ, and (c) RC 


\subsection{Results of proposed system design using $75 \mathrm{~km}$ fiber length}

By looking to the diagrams of Figures 6, 7, and 8, it is clearly showing that the results obtained for a $75 \mathrm{~km}$ transmitting are having the same conclusion for the $100 \mathrm{~km}$ distance. Figure 6 is showing that NRZ signal having the best $\mathrm{Q}$ factor comparing to the $\mathrm{RZ}$ and $\mathrm{RC}$ signals. Looking to Figure 7 is also showing the eye opening for NRZ is wider than for RZ and RC which means that using NRZ as a modulation signal is giving the best performance than using the RZ and RC. Figure 8 is giving the same finding that was from Figure 5, the Oscilloscope Visualizer is showing that the NRZ modulation signal is giving a higher signal amplitude power to noise amplitude power than the other two testing types (RZ and RC) modulation signals.

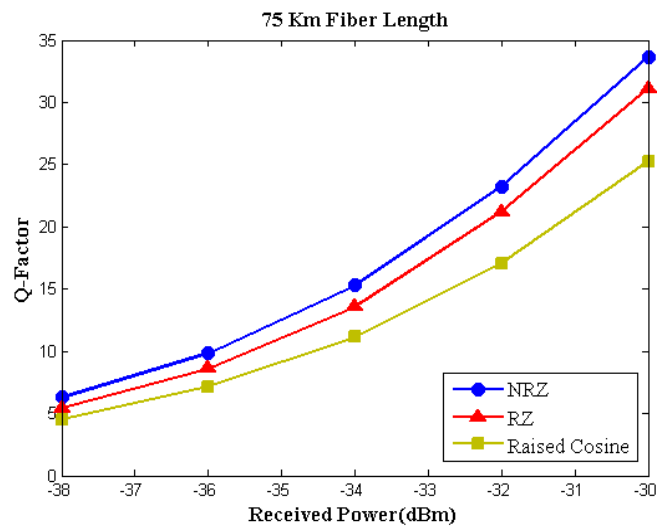

Figure 6. Q factor versus received power at different pulse shape

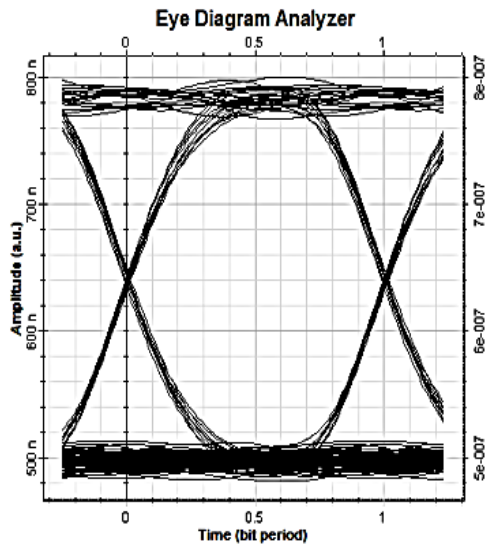

(a)

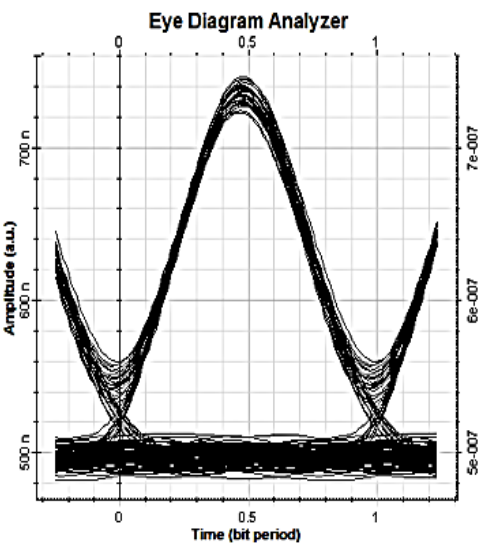

(b)

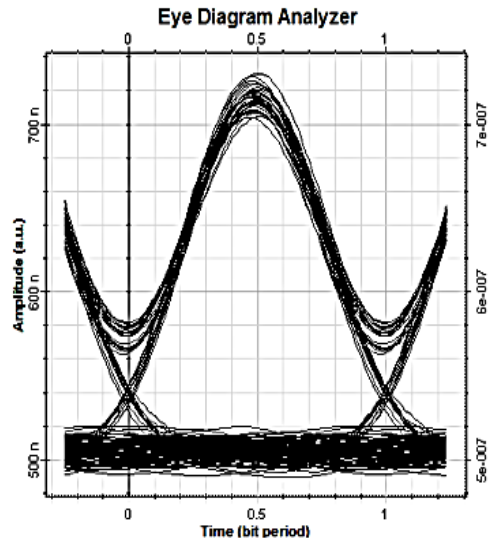

(c)

Figure 7. Optimum Eye diagram performance of $75 \mathrm{~km}$ length for (a) NRZ, (b) RZ, and (c) Raised cosine

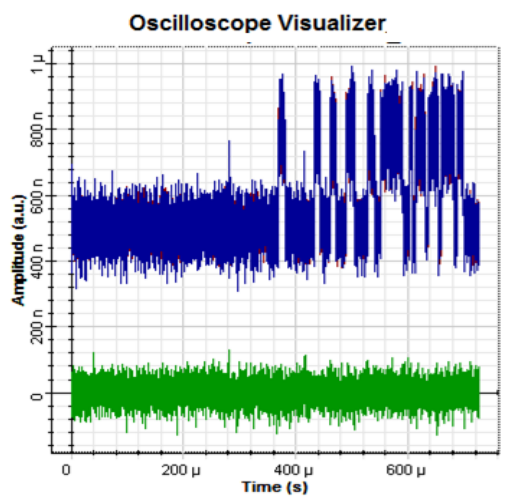

(a)

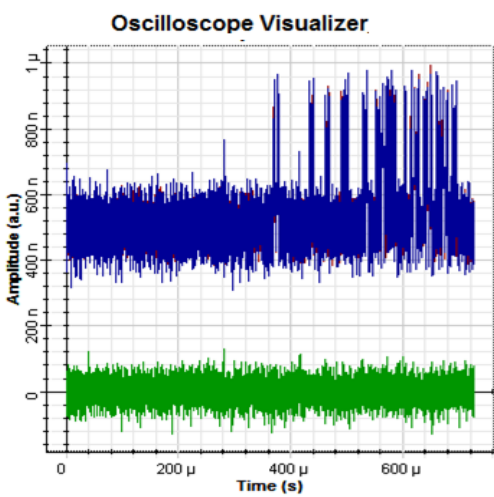

(b)

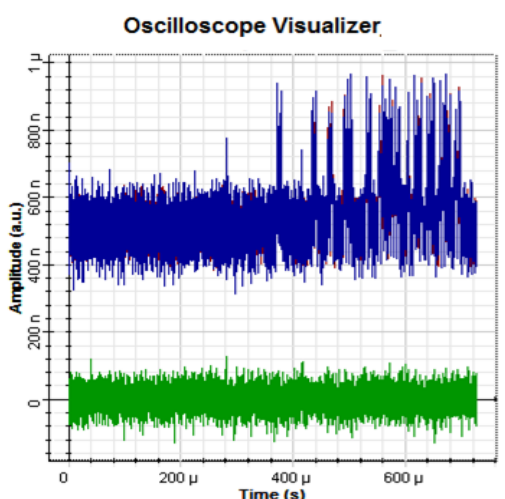

(c)

Figure 8. Signal output using oscilloscope visualizer of $75 \mathrm{~km}$ for (a) NRZ, (b) RZ, and (c) Raised cosine 


\section{CONCLUSION}

The transmission of audio signal through Optical fiber system was designed and performed for first time using Co-Simulation of MATLAB integrated with OptiSystem14 ${ }^{\mathrm{TM}}$. The system was tested for different types of pulse shapes (NRZ, RZ, and RC) modulation signals and for different distances $100 \mathrm{~km}$ and $75 \mathrm{~km}$ of fibers lengths. Different parameters were studied and analyzed and the system was found to be having the best performance with NRZ modulation signal comparing to the other two (RZ and RC) for all three parameters and both distances. The $\mathrm{Q}$ factor, which is an efficiency indicator, is more when NRZ signal is used as a modulation signal 12 for a $-35 \mathrm{dBm}$ received power while it is 10 for $\mathrm{RZ}$ and 8 for $\mathrm{RC}$ at the same power for a $100 \mathrm{~km}$ of transmitting distance. The eye opening is also wider for NRZ than that for $\mathrm{RZ}$ and $\mathrm{RC}$, which means that NRZ modulation pulse, is more resistive to noise. For a $75 \mathrm{~km}$ fiber transmitting distance, the conclusion has no difference than that for a $100 \mathrm{~km}$ distance. The eye opening in case of using NRZ as a modulation signal is wider than using other two types (RZ and RC). The Oscilloscope Visualizer is showing that the use of NRZ is having more confident than the use of RZ and RC because it is having a higher amplitude, which means that the ones and zeros of the received signals could be easily, differentiated when NRZ modulation signal is used comparing to the other two tested modulation signals (RZ and RC).

\section{ACKNOWLEDGEMENTS}

This work was implemented in the laboratories of our Electrical Engineering Department of the University of Babylon/Engineering College. Our regards and thankful to the staff, department's head and the college dean for the help and support.

\section{REFERENCES}

[1] B. N. Mohapatra, "Audio transmitter and receiver system using fiber optic cable," International Journal of Emerging Technologies in Engineering Research, vol. 5, no. 5, May 2017.

[2] G.P. Agrawal, "Fiber Optic Communication Systems," John Wiley \& Sons, New York, 2002

[3] C. C. Yang, "Code space enlargement for hybrid fiber radio and baseband OCDMA PONs," Journal of Light wave Technology, vol. 29, no. 9, pp. 1394-1400, 2011.

[4] S. Zeadally and L. Zhang, "Enabling gigabit network access to end users," Proceedings of the IEEE, vol. 92, no. 2, pp. 340-353, 2004.

[5] R. Ramaswami, K. Sivarajan, and G. Sasaki, "Optical networks: a practical perspective," Morgan Kaufmann, 2009.

[6] G. Farrell, Q. Wang, and W. Yan, "Investigation on single mode-multil mode-single mode fiber structure," Journal of Light Technology, vol. 26, no. 5, 2008.

[7] O. Ziemann, J. Krauser, P. E. Zamzow, and W. Daum, "POF-polymer optical fibers for data communication," Springer Science \& Business Media, 2013.

[8] J. Zubia and J. Arrue, "Plastic optical fibers: An introduction to their technological processes and applications," Optical Fiber Technology, vol. 7, no. 2, pp. 101-140, 2001.

[9] T. Morioka, Y. Awaji, R. Ryf, P. Winzer, D. Richardson, and F. Poletti, "Enhancing optical communications with brand new fibers," IEEE Communications Magazine, vol. 50, no. 2, pp. s31-s42, 2012.

[10] A.K. Sharma, R.K. Sinha, S.K. Sharma, S.K. Arya, R.S. Kaler, "Improved small signal analysis for dispersive optical fiber communication systems," J. Opt. Commun., pp. 1-6, 2004.

[11] H. J. Abed, N. M. Din, M. H. Al-Mansoori, H. A. Fadhil, and F. Abdullah, "Recent four-wave mixing suppression methods," Optik, vol. 124, no. 15, pp. 2214-2218, 2013.

[12] H. J. Abd, M. H. Al-Mansoori, N. M. Din, F. Abdullah, and H. A. Fadhil, "Priority-based parameter optimization strategy for reducing the effects of four-wave mixing on WDM system," Optik, vol. 125, no. 1, pp. 25-30, 2014.

[13] H. Abd, et al., "Four-wave mixing crosstalk suppression based on the pairing combinations of differently linearpolarized optical signals," The Sci. World J., vol. 2014, pp. 1-10, 2014,

[14] H. J. Abd, M. H. Al-Mansoori, N. M. Din, F. Abdullah, and H. A. Fadhil, "Four-wave mixing reduction technique based on smart filter approach," International Journal of Electronics, vol. 102, no. 6, pp. 1056-1070, 2015.

[15] N.M. Ridzuan, M.F.L. Abdullah, M.B. Othman and M.B. Jaafar, "A carrier less amplitude phase (CAP) modulation format: Perspective and prospect in optical transmission system," International Journal of Electrical and Computer Engineering (IJECE), vol. 8, no. 1, pp. 585-595, Feb. 2018.

[16] N. Salim, H.J. Abd, A.N. Aljamal, and A.H. Jaber, "Four-wave mixing suppression method based on odd-even channels arrangement strategy," Progress In Electromagnetics Research, vol. 66, pp. 163-172, 2018.

[17] B. Patnaik and P.K. Sahu, "Novel QPSK modulation for DWDM free space optical communication system," 21st Annual Wireless and Optical Communications Conference (WOCC), pp. 170-175, 2012.

[18] M.A. Shah, S. Abd Latiff, and Riaz T., "Performance measurement of free-space optical $980 \mathrm{~nm}$ channel using multiple sets of environmental conditions," Wireless Personal Communication, vol. 85, no. 2, pp. 345-357, 2015.

[19] V.G.A. Carneiro, G.K. Rodrigues, M. Thereza, and M.R. Giraldi, "Performance analysis of a 2D double hard-limited OCDMA system over FSO link under strong turbulence for defense applications," Military Communications Conference, MILCOM, pp. 1-6, 2012. 
[20] R.A. Fayadh, M.K. Wali, and M.F. Bonneya, "Establishment network by using FSO link based on MD code for hybrid SCM-SAC-OCDMA wireless system," International Journal of Electrical and Computer Engineering (IJECE), vol. 8, no. 6, pp. 5107-5117, Dec. 2018.

[21] A.R. Chraplyvy, "Limitations on lightwave communications imposed by optical fiber nonlinearities," J. Lightw. Technol., vol. 8, no. 10, pp. 1548-1557, 1990

[22] X. Feng, F. Poletti, A. Camerlingo, F. Parmigiani, P. Petropoulos, P. Horak, G.M. Ponzo, M. Petrovich, Jindan Shi, W.H. Loh, and D.J. Richardson, "Dispersion controlled highly nonlinear fibers for all-optical processing at telecoms wavelengths," Optic. Fiber Technology, vol. 16, no. 6, pp. 378-391, 2010.

[23] T. Taniguchi, N. Sakurai, H. Kimura, K. Kumozaki, "Experimental demonstration of tolerance to FWM in crosstalk in wavelength-swept WDM access systems," IECE Electronics Express, vol. 6, no. 16, pp. 1180-1185, 2009.

[24] P. J. Winzer and R. J. Essiambre, "Advanced optical modulation," Proc. ECOC, Rimini, Italy, Invited paper Th2.6.1, pp. 1002-1003, 2003.

[25] A. Gnauck and P. Winzer, "Optical phase-shift-keyed transmission," Journal of Lightwave Techno., vol. 23, no. 1, pp. 115-130, 2005.

[26] R.S. Kaler, A.K. Sharma, and T.S. Kamal, "Simulation results for four wave mixing in an optical fiber near zero dispersion wavelength," J. Electron.Teclnol., vol. 85, pp. 31-36, Jul. 2004.

[27] U. Stanley, V.M. Olu, C. Ochonogor, A. Peter, and A. Francis, "Experimental analysis of cable distance effect on signal attenuation in single and multimode fiber optics," International Journal of Electrical and Computer Engineering (IJECE), vol. 8, no. 3, pp. 1577-1582, Jun. 2018

[28] M. Aljanabi, "Resonance frequency analysis of laser optical fiber based on micro cantilever," International Journal of Electrical and Computer Engineering (IJECE), vol. 9, no. 4, pp. 3090-3099, Aug. 2019.

[29] K.S. Alaoui, J. Foshi, and Y. Zouine, "Radio over fiber system based on a hybrid link for next generation of optical fiber communication," International Journal of Electrical and Computer Engineering (IJECE), vol. 9, no. 4, pp. 2571-2577, Aug. 2019.

[30] A. Shaban, M. O. Abed, E. A. Hussein, and H. J. Abd, "Durability of modulation technique to channel crosstalk effect in optical communication link via applying high data rate," International Journal of Electrical and Computer Engineering (IJECE), vol. 10, no. 1, pp. 5107-5117, 2019.

[31] T.H. Abd, S.A. Aljunid, H.A. Fadhil, R.A. Ahmad, and N.M. Saad, "Development of a new code family based on SAC-OCDMA system with large cardinality for OCDMA network," Optical Fiber Technology, vol. 17, no. 4, pp. 273-280, 2011.

\section{BIOGRAPHIES OF AUTHORS}

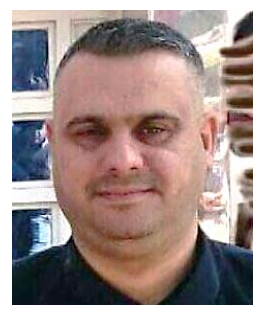

Associate professor Dr. Haider .J. Abd earned his Ph.D. in the field of Electrical Engineering from university of Tenaga Nasional, Malaysia, 2014. He has more than ten years of experience in teaching. He completed his MSc, in Electrical Engineering, university of Baghdad, Baghdad, Iraq, 2005. His research interests includes optical fiber communication, Wireless communication, Digital signal processing, smart systems, Biomedical Engineering, Maglev system and Adaptive control system.

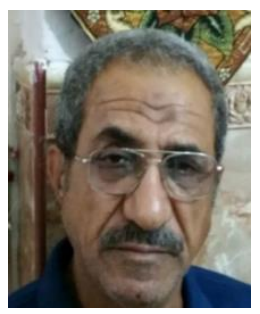

Abdulrasul A. Al-Hayder earned his M.sc in the field of Electrical Engineering from university of technology, Iraq, 1990. He has more than five years of experience in teaching. He completed his BSc in Electrical Engineering, university of technology, Baghdad, Iraq, 1981. His research interests include communication, wavelet transform, electronic system design and Quantum communication.

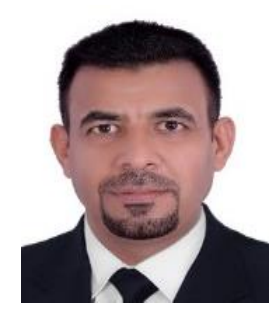

Ahmed Samawi Alkhafaji earned his M.sc in the field of Electrical and Electronic Engineering from Eastern Mediterranean University, Turkey/North Cyprus2013. He has more than five years of experience in teaching. He completed his B.Sc in Electrical Engineering, university of technology, Baghdad, Iraq, 2001. His research interests includes power electronics, control power system, electronic system design and predictive current control modeling. 